\title{
Effective one-dimensionality of universal ac hopping conduction in the extreme disorder limit
}

\author{
Jeppe C. Dyre and Thomas B. Schrøder \\ Department of Mathematics and Physics, Roskilde University, Postbox 260, DK-4000 Roskilde, Denmark
}

(Received 23 February 1996; revised manuscript received 23 August 1996)

\begin{abstract}
A phenomenological picture of ac hopping in the symmetric hopping model (regular lattice, equal site energies, random energy barriers) is proposed according to which conduction in the extreme disorder limit is dominated by essentially one-dimensional "percolation paths." Modeling a percolation path as strictly one dimensional with a sharp jump rate cutoff leads to an expression for the universal ac conductivity that fits computer simulations in two and three dimensions better than the effective medium approximation. [S0163-1829(96)06745-8]
\end{abstract}

While ordered solids show no frequency dependence of their conductivity at frequencies below phonon frequencies, disordered solids are characterized by ac conductivity that varies as an approximate power law of frequency. ${ }^{1-6}$ The exponent is usually less than, but often close to, 1. As the frequency goes to zero the conductivity becomes frequency independent. These features are observed universally for electronically conducting disordered solids like amorphous semiconductors, ${ }^{1,2,4,7}$ polymers, ${ }^{8,9}$ doped-crystalline semiconductors at helium temperatures ${ }^{10}$ (where the random positions of the dopant atoms become important), or hightemperature superconductors above $T_{c},{ }^{11}$ as well as for ionically conducting disordered solids like glasses or polymers. $2,3,5,6$

This paper deals with ac hopping conduction in disordered solids, but before proceeding we note that the study of stochastic motion in disordered systems ("rugged energy landscapes") is relevant in a number of other contexts. ${ }^{12-14}$ Examples include models for protein dynamics, ${ }^{15-18}$ flow of viscous liquids close to the glass transition, ${ }^{19,20}$ diffusion in random flows, ${ }^{21}$ or rate processes controlled by the anomalous diffusion of reactants. ${ }^{22-24}$ Diffusion in a disordered system is characterized by a mean-square displacement that at short times varies more rapidly than expected from extrapolating the long-time linear "Einsteinian" time dependence. If the mean-square displacement in an axis direction $i$ as a function of time is denoted by $\left\langle\Delta X_{i}^{2}(t)\right\rangle$, the frequency-dependent diffusion constant is defined ${ }^{25}$ by [where $s$ denotes the "Laplace" (imaginary) frequency, $s=i \omega]$

$$
D(s)=\frac{s^{2}}{2} \int_{0}^{\infty}\left\langle\Delta X_{i}^{2}(t)\right\rangle e^{-s t} d t .
$$

For diffusion in an ordered structure, where $\left\langle\Delta X_{i}^{2}(t)\right\rangle=2 D t$, one has $D(s)=D$. According to the fluctuation-dissipation theorem, if the diffusing particle carries a charge, $D(s)$ is proportional to the frequencydependent conductivity $\sigma(s) .{ }^{26}$ Consequently, all results derived below for the normalized frequency-dependent conductivity $\widetilde{\sigma}=\sigma(s) / \sigma(0)$ are valid for the normalized frequency-dependent diffusion constant $\widetilde{D}=D(s) / D(0)$.

We consider hopping of charge carriers on a regular lattice with equal site energies and random nearest-neighbor jump rates (model A of Ref. 14). This model for ac conduction in disordered solids has been studied extensively during the last 15 years. ${ }^{26-33}$ If the jump rates are taken to be thermally activated with randomly varying activation energies, the limit of extreme disorder may be studied by letting the temperature go to zero. It has recently been $\operatorname{shown}^{34}$ that in this limit the ac conductivity in suitably scaled units becomes universal in more than one dimension, i.e., independent of temperature and of the activation energy probability distribution $p(E)$. The existence of universality was predicted by the effective medium approximation (EMA) and confirmed by computer simulations in two dimensions. If $\tilde{s}$ is the scaled dimensionless Laplace frequency, the EMA universality equation $^{34}$ is

$$
\widetilde{\sigma} \ln \widetilde{\sigma}=\widetilde{s} .
$$

This equation ${ }^{35}$ was originally derived by Bryksin for the model of electrons tunneling between randomly localized positions. $^{28,36}$

While the existence of universality was confirmed by computer simulations in two dimensions, the onset of ac conduction turned out to be smoother than predicted by Eq. (2). ${ }^{34}$ The EMA is thus qualitatively correct by predicting universality in the extreme disorder limit, but quantitatively inaccurate. This is perhaps not surprising. After all, the EMA replaces the disordered solid by an "effective" homogeneous solid with characteristics determined by a selfconsistency condition. Such an ordered medium cannot $a$ priori be expected to accurately represent conduction in the extreme disorder limit. ${ }^{37}$

It is well known that dc and low-frequency ac hopping at extreme disorder is dominated by percolation effects, i.e., mainly take place on the percolation cluster. ${ }^{28,37-39}$ The percolation cluster is a complicated object with fractal dimension equal to 1.9 and 2.5 in two and three dimensions, respectively. ${ }^{21}$ Removing "dead ends" (contributing little to the low-frequency conductivity) from the percolation cluster leaves us with the "'backbone,', which has fractal dimension equal to 1.6 and 1.7 in two and three dimensions, respectively. ${ }^{21}$ The backbone contains loops, but at low tem- 

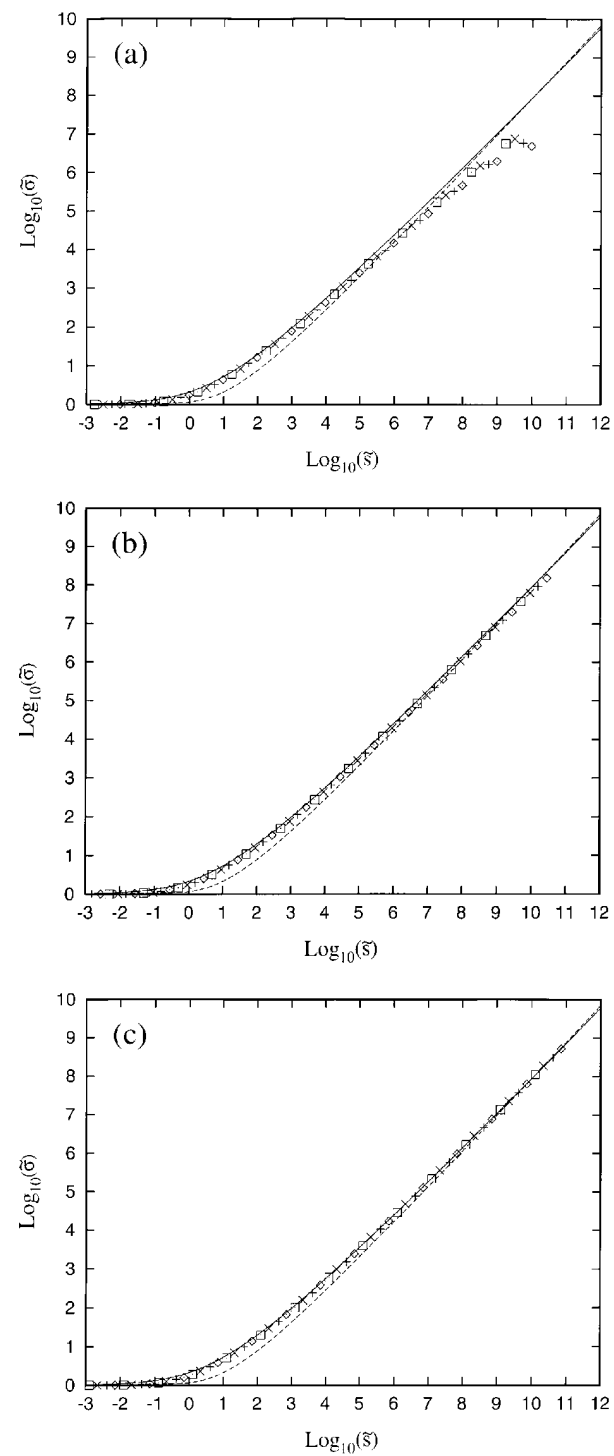

FIG. 1. log-log plot of computer simulations (symbols) of lowtemperature ac conductivity in three dimensions at real Laplace frequencies $s(s=i \omega)$ for four different activation energy probability distributions, compared to the PPA [Eq. (11), solid curve] and to the zero-temperature limit of the EMA universality equation (2), dashed curve. The dimensionless Laplace frequency $\tilde{s}$ is defined by Eq. (10)—however, a further (minor) empirical rescaling was applied to focus exclusively on the shape of the conductivity curve; $\widetilde{\sigma}$ is defined by $\widetilde{\sigma}=\sigma(\widetilde{s}) / \sigma(0)$. The jump rates are given by $\Gamma=\Gamma_{0} \exp (-\beta E)$, where $\beta$ is the inverse temperature and the activation energy $E$ is chosen randomly according to the following probability distributions (Ref. 40): asymmetric Gaussian $\left[p(E) \propto \exp \left(-E^{2} / 2\right)\right.$, $0<E<\infty](\times)$, Cauchy $\left[p(E) \propto 1 /\left(1+E^{2}\right), 0<E<\infty\right](+)$, exponential $[p(E)=\exp (-E), 0<E<\infty](\diamond)$, and box $[p(E)=1,0<E<1](\square)$. To speed up the calculations all jump rates with activation energy larger than $E_{c}+K / \beta$ were set to zero, where $E_{c}$ is defined from the bond percolation threshold in Eq. (3). By varying the factor $K$ the maximum errors introduced by this approximation were estimated to be below $1 \%$ for both $\sigma(0)$ and $\widetilde{\sigma}(\widetilde{s})$ for the $K=6.4$ used for the data presented here. In terms of the dimensionless inverse temperature $\widetilde{\beta}=\beta / p\left(E_{c}\right)$ the figure shows data for 100 averages of simulations of cubic lattices with linear dimension $N$ where (a) $\widetilde{\beta}=80(N=29),(b) \widetilde{\beta}=160(N=54)$, and (c) $\widetilde{\beta}=320(N=100)$ [by varying $N$, the maximum errors introduced by using finite lattices are estimated to be below $30 \%$ and $5 \%$ for $\sigma(0)$ and $\widetilde{\sigma}(\widetilde{s})$, respectively]. This figure shows that the ac conductivity becomes universal at low temperatures and that the PPA gives a better fit to data than the EMA universality equation. peratures one branch of a loop usually has a much higher conductivity than others. The dimension of the actual conduction paths must thus be lower than the dimension of the backbone. Given these arguments, we now make the simplest possible assumption by regarding the conducting paths as one dimensional. This approach, which works well for impedance networks in the extreme disorder limit, ${ }^{40}$ is referred to as the "percolation path approximation", (PPA).

It should be stressed that the PPA is an approximation that builds on a highly phenomenological picture of conduction in the extreme disorder limit. In a sense, the PPA may be viewed as being complementary to the EMA: In the EMA the disordered medium is replaced by a homogeneous medium of the same dimension (determined by a selfconsistency condition), while in the PPA the disordered medium is replaced by one dimensional conduction paths (determined by percolation arguments).

To summarize, in the PPA conduction takes place via " "percolation"' paths that have two characteristics: They are strictly one dimensional and they only involve activation energies up to the "percolation energy" $E_{c}$. This quantity, which is known to be the activation energy of the dc conductivity, ${ }^{41,42}$ is defined from the bond percolation threshold $p_{c}$ by

$$
p_{c}=\int_{0}^{E_{c}} p(E) d E .
$$

The purpose of this paper is to show that the PPA approach to ac conduction in the extreme disorder limit, reminiscent of the "conducting path model,",43,44 gives a good representation of computer simulations. We thereby indirectly confirm the recent findings of Brown and Esser, ${ }^{45}$ according to which the actual paths of particles in disordered systems become predominantly one dimensional when the disorder is strong.

Unfortunately, the one-dimensional hopping model behind the PPA is not analytically solvable. Below we derive an approximation to the PPA utilizing the one-dimensional EMA, which is known to work very well ${ }^{46}$ [this is confirmed below in Fig. 2(a)]. We then show by computer simulations that this one-dimensional EMA, henceforth identified with the PPA, gives a better representation of the universal lowtemperature ac hopping conductivity in two and three dimensions than Eq. (2) does.

To arrive at the PPA, hopping in one dimension with a sharp activation energy cutoff is addressed [i.e., $p(E)=0$ for $E>E_{c}$ and $\left.p\left(E_{c}\right)>0\right]$. In the "rationalized" unit system where the conductivity for a homogeneous system is equal to the jump rate, ${ }^{29}$ the EMA equation for the ac conductivity $\sigma(s)$ in one dimension ${ }^{26,29,33,34,46-50}$ is (where $\Gamma$ is the jump rate and the brackets denote an average over the jump rate probability distribution)

$$
\langle(\Gamma-\sigma) /[\sigma+(1-s \widetilde{G})(\Gamma-\sigma)]\rangle=0 .
$$

Here, $\widetilde{G}$ is the diagonal element of the Green's function for a random walk on a one-dimensional lattice with uniform jump rate $\sigma$ (the "effective medium"'); $s \widetilde{G}$ is given ${ }^{34,46,50}$ by

$$
s \widetilde{G}=(1+4 \sigma / s)^{-1 / 2} .
$$


We are only concerned with relatively low frequencies where $s \widetilde{G} \ll 1$. To lowest order in $s \widetilde{G}$, Eq. (4) implies ${ }^{51}$

$$
1 / \sigma=\langle 1 /(\Gamma+s \widetilde{G} \sigma)\rangle .
$$

The right-hand side may be expanded as a power series in $s \widetilde{G} \sigma$, leading to

$$
\frac{1}{\sigma}=\sum_{n=0}^{\infty}(-s \widetilde{G} \sigma)^{n}\left\langle\Gamma^{-(n+1)}\right\rangle .
$$

Since $\Gamma=\Gamma_{0} \exp (-\beta E)$, where $\beta$ is the inverse temperature, the average $\left\langle\Gamma^{-(n+1)}\right\rangle$ is easily evaluated in the lowtemperature limit: If $\widetilde{\beta}=\beta / p\left(E_{c}\right)$, one finds to leading order in $1 / \widetilde{\beta}$

$$
\left\langle\Gamma^{-(n+1)}\right\rangle=\int_{0}^{E_{c}} \Gamma_{0}^{-(n+1)} e^{(n+1) \beta E} p(E) d E=\frac{\Gamma\left(E_{c}\right)^{-(n+1)}}{(n+1) \widetilde{\beta}} .
$$

When this is substituted into Eq. (7) the following is obtained:

$$
\frac{1}{\sigma}=\sum_{n=0}^{\infty}(-s \widetilde{G} \sigma)^{n} \frac{\Gamma\left(E_{c}\right)^{-(n+1)}}{(n+1) \widetilde{\beta}}=\frac{1}{\widetilde{\beta} s \widetilde{G} \sigma} \ln \left[1+\frac{s \widetilde{G} \sigma}{\Gamma\left(E_{c}\right)}\right] .
$$

Letting $s$ go to zero we find $\sigma(0)=\widetilde{\beta} \Gamma\left(E_{c}\right)$. Introducing the dimensionless Laplace frequency

$$
\widetilde{s}=\left[\widetilde{\beta}^{2} / 4 \sigma(0)\right] s,
$$

one finds that, whenever $s \widetilde{G} \ll 1$, Eq. (5) implies $\widetilde{\beta} s \widetilde{G}=\sqrt{\widetilde{s} / \widetilde{\sigma}}$ [where as in Eq. (2) $\widetilde{\sigma}=\sigma / \sigma(0)]$. Substituting this and $\Gamma\left(E_{c}\right)=\sigma(0) / \widetilde{\beta}$ into Eq. (9) finally leads to the PPA expression

$$
\sqrt{\widetilde{\sigma}} \ln [1+\sqrt{\widetilde{s} \widetilde{\sigma}}]=\sqrt{\widetilde{s}} .
$$

Due to the factor $\widetilde{\beta}$ in $\widetilde{\beta} s \widetilde{G}=\sqrt{\widetilde{s} / \widetilde{\sigma}}$, as the temperature is lowered towards zero the condition $s \widetilde{G} \ll 1$ is obeyed in a wider and wider range of dimensionless frequencies. Note that Eqs. (10) and (11) imply that the frequency marking the onset of ac conduction in real units has roughly the same activation energy as the dc conductivity. ${ }^{31} \mathrm{We}$ also note that Eq. (11) implies that the equilibrium mean-square displacement at short times varies proportionally to $1 / \mathrm{ln}^{2}\left(t^{-1}\right) .^{52}$

We have carried out computer simulations of lowtemperature ac hopping in one, two, and three dimensions using the Fogelholm algorithm ${ }^{53}$ to reduce the ac MillerAbrahams electrical equivalent circuit of hopping ${ }^{54-56}$ according to a recently proposed scheme. ${ }^{34}$ While standard Monte Carlo techniques can hardly be used for simulations at inverse temperatures above $\widetilde{\beta}=20,{ }^{45,57}$ the new scheme can be used at much lower temperatures (in our simulations up to inverse temperatures of $\widetilde{\beta}=320$ ). Figure 1 shows the results of our simulations of low-temperature ac hopping in three dimensions at real Laplace frequencies. Results are shown for averages of 100 simulations of the ac conductivity for four different activation energy probability distributions at the following inverse temperatures: (a) $\widetilde{\beta}=80$, (b) $\widetilde{\beta}=160$, and (c) $\widetilde{\beta}=320$. The solid curve is the PPA [Eq. (11)] and
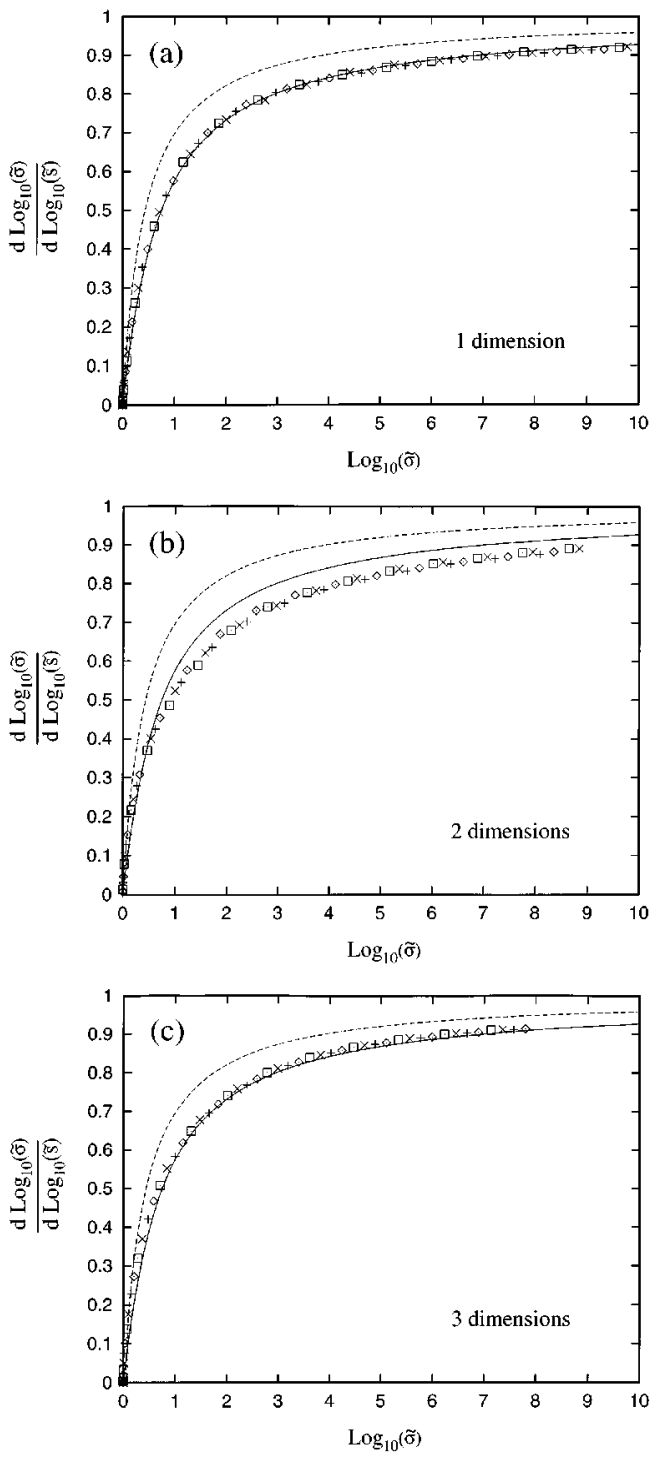

FIG. 2. The slope of the log-log plot of $\widetilde{\sigma}(\widetilde{s})$ at real Laplace frequencies, $d \log _{10}(\widetilde{\sigma}) / d \log _{10}(\widetilde{s})$, as function of $\log _{10}(\widetilde{\sigma})$ for simulations at the dimensionless inverse temperature $\widetilde{\beta}=320$ in (a) one dimension (100 averages of 8192 point lattices), (b) two dimensions (30 averages of $880 \times 880$ lattices), and (c) three dimensions (100 averages of $100 \times 100 \times 100$ lattices) for the four activation energy probability distributions of Fig. 1. The simulations in one dimension were carried out with a sharp activation energy cutoff at $E=1$ in order to show the validity of the one-dimensional EMA used in deriving Eq. (11). In two and three dimensions, as in Fig. 1, the smallest jump rates were set to zero to speed up the simulations. The simulations are compared to the predictions of the PPA [solid curve, Eq. (11)] and to the EMA universality equation [dashed curve, Eq. (2)]. Both the EMA and the PPA predict that the slope of the log-log plot goes to 1 as $\widetilde{s} \rightarrow \infty$, as seen in experiment (Ref. 1). However, the PPA works better than the EMA in two dimensions and much better than the EMA in three dimensions.

the dashed curve is the EMA universality equation (2). Empirical rescalings of the frequency were allowed in order to focus only on the shape of the conductivity curves. Figure 1 shows that universality is approached as the temperature goes to zero and that the PPA gives a quite good fit to the universal ac conductivity in three dimensions.

The universality may be studied without use of empirical rescalings by plotting the slope of the log-log plot, $d \log _{10}(\widetilde{\sigma}) / d \log _{10}(\widetilde{s})$, as a function of $\log _{10}(\widetilde{\sigma})$. This is done in Fig. 2 for the same activation energy probability distributions as in Fig. 1 for $\widetilde{\beta}=320$ data from computer simulations 
in one, two, and three dimensions. The solid curve is the PPA prediction and the dashed curve is the EMA universality equation. The simulations in one dimension were for systems with a sharp activation energy cutoff; these simulations were carried out to ensure that Eq. (11) does indeed give an accurate representation of this situation. The real subject of interest is ac conduction in two and three dimensions without any activation energy cutoff [Figs. 2(b) and 2(c)]. In both dimensions the PPA works better than the EMA universality equation.

Considering the simplicity of the phenomenological picture behind the PPA, the agreement with computer simulations in three dimensions is striking. We take our results as an indication that, at least in three dimensions, lowfrequency conduction at extreme disorder in fact is dominated by essentially one-dimensional conduction paths. This makes sense in light of the "nodes-links" $\operatorname{model}^{58,59}$ for the infinite network just above the percolation threshold ( $\mathrm{dc}$ and low-frequency ac conduction are known to take place on this network ${ }^{28}$ ): According to this model, the backbone of the percolation cluster comprises links (quasi-one-dimensional strings) and nodes (intersection of links). Possibly, the PPA works well because it models the ac response of a single link. Note that "blobs" on the percolation cluster, as in the more realistic "nodes-links-blobs" model, ${ }^{58,59}$ are expected to be unimportant in the extreme disorder limit, since usually one branch of the blob has a much higher conductivity than others.

The problem of ac hopping in the extreme disorder limit is still far from fully understood. This is indicated by the deviation between the PPA and computer simulations in two dimensions, where the PPA according to the arguments involving the fractal dimension of the backbone should work slightly better than in three dimensions and not much worse ${ }^{60}$ Clearly, more work is needed before a genuine understanding of universal ac hopping in the extreme disorder limit is arrived at. Thus, ac hopping in more than three dimensions should be simulated. Also, the actual paths of the particles should be traced out to see whether they are indeed almost one dimensional. Finally, a closer investigation of the analyticity properties of the universal ac conductivity is needed to throw light on whether the prediction of the PPA (that $\widetilde{\sigma}-1 \propto \widetilde{s}^{-1 / 2}$ for $\widetilde{s} \rightarrow 0$ ) is really obeyed.
${ }^{1}$ A. K. Jonscher, Nature 267, 673 (1977); Universal Relaxation Law (Chelsea Dielectric, London, 1996).

${ }^{2}$ A. E. Owen, J. Non-Cryst. Solids 25, 372 (1977)

${ }^{3}$ M. D. Ingram, Phys. Chem. Glasses 28, 215 (1987).

${ }^{4}$ S. R. Elliott, Physics of Amorphous Materials, 2nd ed. (Longman Scientific, London, 1990).

${ }^{5}$ C. A. Angell, Chem. Rev. 90, 523 (1990).

${ }^{6}$ K. Funke, Prog. Solid State Chem. 22, 111 (1993).

${ }^{7}$ A. R. Long, Adv. Phys. 31, 553 (1982).

${ }^{8}$ A. R. Blythe, Electrical Properties of Polymers (Cambridge University Press, London, 1979).

${ }^{9}$ F. Kremer et al., Makromol. Chem. Macromol. Symp. 37, 115 (1990).

${ }^{10}$ M. Pollak and T. H. Geballe, Phys. Rev. 122, 1742 (1961).

${ }^{11}$ S. Mollah et al., J. Appl. Phys. 74, 931 (1993).

${ }^{12}$ P. G. Wolynes, Acc. Chem. Res. 25, 513 (1992).

${ }^{13}$ S. A. Kauffman, The Origins of Order (Oxford University Press, Oxford, 1993).

${ }^{14}$ D. L. Stein and C. M. Newman, Phys. Rev. E 51, 5228 (1995).

${ }^{15} \mathrm{H}$. Frauenfelder et al., Science 254, 1598 (1991).

${ }^{16}$ H. Frauenfelder and P. G. Wolynes, Phys. Today 47(2), 58 (1994).

${ }^{17}$ J. D. Bryngelson et al., Proteins Struct. Funct. Genet. 21, 167 (1995).

${ }^{18}$ Yu. A. Berlin et al., Chem. Phys. 200, 369 (1995).

${ }^{19}$ R. Richert and H. Bässler, J. Phys. Condens. Matter 2, 2273 (1990).

${ }^{20}$ J. C. Dyre, Phys. Rev. B 51, 12276 (1995).

${ }^{21}$ M. B. Isichenko, Rev. Mod. Phys. 64, 961 (1992).

${ }^{22}$ A. Plonka, Time-Dependent Reactivity of Species in Condensed Media, Lecture Notes in Chemistry (Springer, Berlin, 1986).

${ }^{23}$ Yu. A. Berlin, Mol. Cryst. Liq. Cryst. 228, 93 (1993).

${ }^{24}$ A. Plonka, Annu. Rep. Prog. Chem. Sec. C 91, 107 (1994).

${ }^{25}$ H. Scher and M. Lax, Phys. Rev. B 7, 4491 (1973).

${ }^{26}$ T. Odagaki and M. Lax, Phys. Rev. B 24, 5284 (1981).

${ }^{27}$ S. Alexander et al., Rev. Mod. Phys. 53, 175 (1981).

${ }^{28} \mathrm{H}$. Böttger and V. V. Bryksin, Hopping Conduction in Solids (AkademieVerlag, Berlin, 1985).

${ }^{29}$ J. W. Haus and K. W. Kehr, Phys. Rep. 150, 263 (1987).

${ }^{30}$ G. A. Niklasson, J. Appl. Phys. 62, R1 (1987).

${ }^{31}$ J. C. Dyre, J. Appl. Phys. 64, 2456 (1988).

${ }^{32}$ J.-P. Bouchaud and A. Georges, Phys. Rep. 195, 127 (1990).

${ }^{33}$ B. D. Hughes, Random Walks and Random Environments (Clarendon, Oxford, 1995).

${ }^{34}$ J. C. Dyre, Phys. Rev. B 49, 11709 (1994); 50, 9692(E) (1994).

${ }^{35}$ Equation (2) predicts that $\widetilde{\sigma}(\widetilde{s})$ is an analytic function. This may seem surprising since at any finite temperature it is well known that $\widetilde{\sigma}(\widetilde{s})$ has nonanalyticities reflecting the long-time tails of the velocity autocorrelation function, nonanalyticities which are also present in the EMA at finite temperatures. However, the EMA universality equation (2) arises in the zero-temperature limit and is thus an example of the fact that a limit of non-analytical functions may very well be analytic.

${ }^{36}$ V. V. Bryksin, Fiz. Tverd. Tela 22, 2441 (1980) [Sov. Phys. Solid State 22, 1421 (1980)].

${ }^{37}$ A. Hunt, J. Non-Cryst. Solids 183, 109 (1995).

${ }^{38}$ I. P. Zvyagin, Phys. Status Solidi B 95, 227 (1979).

${ }^{39}$ B. I. Shklovskii and A. L. Efros, Electronic Properties of Doped Semiconductors (Springer, Berlin, 1984).

${ }^{40}$ J. C. Dyre, Phys. Rev. B 48, 12511 (1993).

${ }^{41}$ V. Ambegaokar et al., Phys. Rev. B 4, 2612 (1971).

${ }^{42}$ B. I. Shklovskii and A. L. Efros, Zh. Eksp. Teor. Fiz. 60, 867 (1971) [Sov. Phys. JETP 33, 468 (1971)]

${ }^{43}$ H. Namikawa, J. Non-Cryst. Solids 18, 173 (1975).

${ }^{44} \mathrm{M}$. Tomozawa, in Treatise on Materials Science and Technology, edited by M. Tomozawa (Academic Press, New York, 1977), Vol. 12, p. 283.

${ }^{45}$ R. Brown and B. Esser, Philos. Mag. B 72, 125 (1995).

${ }^{46}$ V. V. Bryksin, Fiz. Tverd. Tela 22, 2048 (1980) [Sov. Phys. Solid State 22, 1194 (1980)].

${ }^{47}$ S. Summerfield, Solid State Commun. 39, 401 (1981).

${ }^{48}$ I. Webman, Phys. Rev. Lett. 47, 1496 (1981).

${ }^{49}$ B. Movaghar and W. Schirmacher, J. Phys. C 14, 859 (1981).

${ }^{50}$ M. Sahimi et al., J. Chem. Phys. 78, 6849 (1983).

${ }^{51}$ Obtained by putting the dimension equal to one and ignoring the unimportant factor $s \widetilde{G}$ in the denominator on the left-hand side of Eq. (46) in Ref. 34.

${ }^{52}$ J. C. Dyre and J. M. Jacobsen, Chem. Phys. 112 (to be published).

${ }^{53}$ R. Fogelholm, J. Phys. C 13, L571 (1980).

${ }^{54}$ A. Miller and E. A. Abrahams, Phys. Rev. 120, 745 (1960).

${ }^{55}$ M. Pollak, in Proceedings of the Fifth International Conference on Amorphous and Liquid Semiconductors, edited by J. Stuke and W. Brenig Garmisch-Partenkirchen, 1973, (Taylor \& Francis, London, 1974), Vol. 1, p. 127.

${ }^{56}$ S. Summerfield and P. N. Butcher, J. Phys. C 15, 7003 (1982).

${ }^{57}$ I. Avramov et al., Phys. Rev. E 47, 2303 (1993).

${ }^{58} \mathrm{D}$. Stauffer and A. Aharony, Introduction to Percolation Theory, 2nd ed. (Taylor \& Francis, London, 1992).

${ }^{59}$ T. Nakayama et al., Rev. Mod. Phys. 66, 381 (1994).

${ }^{60}$ In view of the "nodes-links-blobs" model, one may speculate that the PPA works relatively poorly in two dimensions because the "blobs" here are much more important than in higher dimensions. In this line of reasoning, there are too many branches of a "blob" in two dimensions that one can argue, as above, that one branch of the blob dominates conduction. 